\title{
MEJORAR LA IDENTIDAD EMPRENDEDORA DE LOS ESTUDIANTES UNIVERSITARIOS: UNA EXPERIENCIA FORMATIVA
}

\author{
IMPROVING THE ENTREPRENEURIAL IDENTITY \\ OF UNIVERSITY STUDENTS:
}

A TRAINING EXPERIENCE

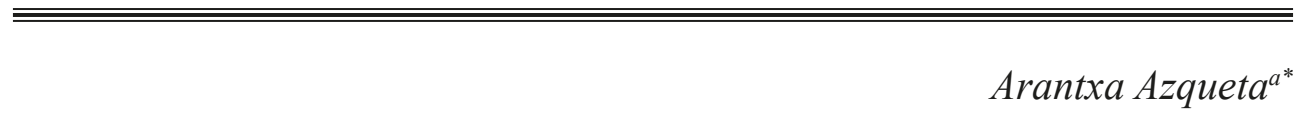

Fechas de recepción y aceptación: 4 de julio de 2021 y 19 de julio de 2021

DOI: https://doi.org/10.46583/edetania_2021.60.930

Resumen: El interés por la educación emprendedora se presenta como una tendencia educativa internacional que ha calado con fuerza en la institución educativa y se ha incorporado en todas las etapas educativas, especialmente en la educación superior. Este artículo presenta la experiencia de la asignatura Formación para el emprendimiento y la innovación que se imparte en la Facultad de Educación y Psicología de la Universidad de Navarra (España). La cuestión clave de esta materia radica en cómo debe ser la educación en emprendimiento para fomentar el crecimiento de la identidad emprendedora de los estudiantes, para que contribuya al crecimiento integral de la persona y no se limite al desarrollo socioeconómico o laboral. El artículo expone, en primer lugar, el enfoque humanista de la asignatura, que busca favorecer el desarrollo de la iniciativa, especialmente de acciones que entrañan cierta dificultad, y que plantea adquirir desafíos y responsabilidades además de promover la actitud de apertura a la realidad, especialmente del entorno próximo. En segundo lugar, se describe la metodología de trabajo de la asignatura que ha empleado el aprendizaje por proyectos. Finalmente, se muestra la experiencia de evaluación interna de la asignatura en la que se combina la metodología cuantitativa, a través de una encuesta a los estudiantes, y la metodología cualitativa, que se sirve de entrevistas semiestructuradas. De esta manera se han podido conocer los puntos fuertes

${ }^{a}$ Facultad de Educación y Psicología. Universidad de Navarra.

* Correspondencia: Universidad de Navarra. Facultad de Educación y Psicología. Campus universitario. 31009 Pamplona. Navarra. España.

E-mail: arantxa.azqueta@unir.net 
y débiles de la asignatura y desarrollar algunas propuestas de mejora, que, tanto a corto como a medio plazo, incrementen la capacidad formativa de la asignatura.

Palabras clave: educación emprendedora, identidad emprendedora, universidad emprendedora, desarrollo personal, aprendizaje por proyectos.

Abstract: Interest in entrepreneurship education is presented as an international educational trend that has strongly entered educational institutions and has been incorporated in all educational stages, especially in higher education. This article presents the experience from a course called Training for Entrepreneurship and Innovation that was taught at the Faculty of Education and Psychology of the University of Navarra (Spain). The key question posed by this course is what kind of entrepreneurship education promotes the entrepreneurial spirit of the students, contributes to the integral growth of the person, and is not limited to their socioeconomic or employment development. First, this article presents the humanistic approach of this course, which seeks to develop personal initiative, especially those actions that involve some difficulty, entail assuming risks and responsibilities, as well as promoting an attitude of openness to reality, especially one's immediate surroundings. Second, the article describes the course's methodology that is project-based learning. Finally, we show the outcomes from the internal evaluation of the course. These combine quantitative methodology, through a survey of students, and qualitative methodology that uses semi-structured interviews. In this way, it has been possible to know the strengths and weaknesses of the course and develop some proposals for improvement, which would increase the formative impact of the course in short and medium terms.

Keywords: entrepreneurship education, entrepreneurial identity, entrepreneurial university, personal development, project-based learning.

\section{INTRODUCCIÓN}

Desde hace unas décadas, el interés por una cultura emprendedora se ha convertido en un objetivo educativo que ha calado con fuerza en las instituciones educativas. Se considera que es un medio para favorecer el cambio de mentalidad que necesita la sociedad europea para adaptarse a los requerimientos del siglo XXI (Consejo Europeo, 2000). En este sentido, la reciente pandemia por la COVID-19 ha puesto de manifiesto la necesidad de la capacidad emprendedora. Toda la población hemos tenido que poner en juego nuestra capacidad de adaptación, de iniciativa, de generación de ideas y resolución de problemas, junto a la responsabilidad ante una situación incierta, nueva y compleja (González-Tejerina y Vieira, 2020). 
El interés por la educación emprendedora se ha desarrollado en todas las etapas educativas desde la educación temprana a la educación superior, aunque es en la etapa universitaria en la que tiene una mayor presencia (Rosendahl, Sloof y Van Praag, 2014; Eurydice, 2016; Colther, Fecci, Cayun, G. y Rojas, 2020). El plan Bolonia ha tenido un efecto positivo en el impulso de la educación emprendedora porque ha propiciado, entre otros, el interés por el aprendizaje no formal, la mejora de los lazos entre la Universidad-empleador y la preocupación por la innovación (European Commission, 2008). Asimismo, entre los objetivos de modernización de las instituciones europeas de educación superior se recomienda el estímulo al desarrollo de las capacidades empresariales, creativas e innovadoras (Comisión Europea, 2011). En este sentido, los rectores de las universidades españolas han señalado la necesidad de que desde las aulas de las universidades se contribuya a la mejora del espíritu innovador y emprendedor de nuestros estudiantes (CRUE, 2018). En esta línea, algunas universidades han creado, dentro de los equipos rectorales, vicerrectorados de empleabilidad y emprendimiento u órganos y oficinas específicas para el fomento de la actividad emprendedora cuya finalidad es favorecer el autoempleo y mejorar las posibilidades de empleabilidad de los estudiantes. La reciente Conferencia Ministerial sobre el Espacio Europeo de Educación Superior, celebrada en noviembre de 2020, ha puesto de manifiesto cómo las universidades deben liderar procesos de mejora integral y transversal de la sociedad en la que resulten claves la generación de conocimiento, la innovación, el diálogo y la conexión con la sociedad (Rome Ministerial, 2020).

Habitualmente, el modelo de universidad emprendedora se asocia con modelos gerenciales de universidad y se caracteriza por la capacidad de generar capital emprendedor. Este tipo de universidad mantiene relaciones fluidas con la empresa y ha desarrollado estructuras internas para transferir conocimientos desde el ámbito investigador universitario al sector empresarial mediante la generación, fundamentalmente, de patentes (Audretsch, 2014; Hervás-Oliver, Boronat y Messana, 2017). Sin embargo, se considera que este modelo tiene algunas carencias, como son la orientación al mercado, la valoración monetaria del conocimiento value for money o la limitación de las relaciones al ámbito Universidad-empresa (Castro, Barrenechea e Ibarra, 2011). A su vez, en el diseño curricular de las asignaturas o de los programas relacionados con el 
emprendimiento prevalece un enfoque que asocia la educación emprendedora con la mejora de la capacidad productiva y económica y la adquisición de las habilidades para la generación de empresas (Bernal Guerrero y Cárdenas, 2017). Asimismo, son numerosas las voces que critican el sesgo economicista de la educación emprendedora y se apunta la necesidad de plantear enfoques más integrales que no ciñan la educación emprendedora a la mejora de las capacidades para la generación de proyectos empresariales (Komulainen, Naskali, Korhonen y Keskitalo-Foley, 2011; Gill, 2014). Al mismo tiempo, algunos estudios empíricos consideran que, en los estudios de ciencias de la educación, la competencia de iniciativa y espíritu emprendedor es una de las competencias menos trabajadas a lo largo de los estudios universitarios y resulta, cuando menos, mejorable (Martínez-Clares, González-Lorente y Rebollo, 2019).

El objetivo principal de este trabajo es dar a conocer la experiencia de la puesta en marcha de la asignatura Formación para el emprendimiento y la innovación impartida en la Facultad de Educación y Psicología de la Universidad de Navarra (España). Se muestra el enfoque, la metodología empleada, cómo se ha implementado, la evaluación de la asignatura por parte de los alumnos a través de una encuesta y entrevistas semiestructuradas y las conclusiones obtenidas. Esto ha permitido conocer los puntos fuertes y débiles de esta materia y desarrollar a futuro aspectos de mejora para aumentar el impacto en su trayectoria personal e incrementar la capacidad formativa de esta manera. A través de esta asignatura se busca despertar y mejorar la capacidad emprendedora de los estudiantes y contribuir a su formación integral. Como es bien sabido, el curso 2020-2021 ha supuesto el reto añadido de adaptación a las nuevas circunstancias provocadas por la pandemia de la COVID-19.

\section{Concepto de EDUCACión EMPRENDEdora}

La investigación académica es unánime al manifestar la dificultad de definir la educación emprendedora (Hytti y O'Gorman, 2004; Eurydice, 2016). Su incorporación en el currículo es aún reciente y su aplicación práctica es heterogénea. El aprendizaje se ha convertido en una cuestión de gobierno, que promueve una racionalidad de lo que se considera educativo, donde tiene mayor peso lo económico y lo técnico-instrumental (Simon y Masschelein, 
2008). Atender a las necesidades de progreso y, en concreto, de la economía resulta insuficiente para considerar algo digno de ser enseñado. Para García del Dujo (2011), el utilitarismo ha impulsado una "pedagogización de la vida" que convierte la educación en un instrumento para alcanzar un fin y una herramienta social para favorecer la modernización de la sociedad, la mejora tecnológica y la sostenibilidad de un modelo social preocupado mayoritariamente por el logro de beneficios económicos. El razonamiento que impulsa esta concepción educativa es la teoría del capital humano (Schultz, 1961) que se completa con la perspectiva de la sociología funcionalista de la realidad, caracterizada por el utilitarismo y que concede la máxima importancia a la innovación, la creación tecnológica y el interés por la capacidad económica de los individuos y de la sociedad (Espejo, 2001). A su vez, el emprendimiento sintetiza algunas de las características del capitalismo cognitivo que convierte al emprendedor en un perfil profesional que se adapta a las nuevas prácticas de flexibilidad, iniciativa, creatividad e innovación que requiere el mercado laboral (Ruiz-Herrero, 2013).

La tarea educativa es una acción dispositiva (Pring, 2016) cuya primera acción es seleccionar lo que se enseña de manera que promueva la formación humana y posibilite el desarrollo de sus potencialidades. Tanto la política y la economía como el sistema educativo nos impulsan hacia el hacer, la eficacia y la eficiencia del mercado. Esto, siendo de interés, no es suficiente. Educar no solo es formar a buenos trabajadores, también supone contribuir a formar a buenas y mejores personas. El interés de la educación emprendedora no solo radica en que es fruto de la exigencia de adaptación a los tiempos, ni que prepara para la vida adulta, sino también en que contribuye, al mismo tiempo, al desarrollo personal, a la mejora y al cambio social. En este sentido, por ejemplo, se considera que posibilita el desarrollo de las capacidades, de acuerdo con el enfoque de desarrollo humano propuesto por Sen (1985) y Nussbaum (2012). Estos autores centran la atención en el individuo y entienden la noción de desarrollo como desarrollo de la persona y no tanto del capital humano que busca aumentar los recursos materiales y las posibilidades para producir. Para Nussbaum (2012), el desarrollo personal constituye lo que las personas pueden verdaderamente hacer o ser y entiende las capacidades como oportunidades para hacer lo que se desea. Desde una perspectiva educativa, poseer una capacidad implica tener una habilidad propia y una posibilidad real para hacer aquello que se quiere. Así pues, el individuo depende, por un lado, 
del potencial interior que tenga y, por otro lado, de las oportunidades que el entorno pueda ofrecerle (Amilburu, Ruiz-Corbella y García-Gutiérrez, 2017).

\section{ENFOQUE DE LA ASIGNATURA}

La cuestión clave de esta materia radica en cómo debe ser la educación emprendedora para fomentar el crecimiento de la identidad emprendedora de los estudiantes, de manera que se contribuya al crecimiento integral de la persona y no se limite al desarrollo socioeconómico o laboral (Azqueta y Naval, 2019). No se trata de reducir la educación emprendedora a la adquisición de conocimientos, habilidades o experiencias emprendedoras, sino que se busca ayudar a pensar, a reflexionar y, como consecuencia, a contribuir a la mejora personal, al mismo tiempo que se promueven valores ciudadanos. Desde el punto de vista conceptual, la asignatura responde a un modelo humanista que distinguen dos dimensiones de la cultura emprendedora. En primer lugar, el desarrollo de la iniciativa y el impulso de acciones que encierran dificultad y requieren la voluntad de tomar responsabilidades y desafíos. En segundo lugar, la actitud de apertura a lo real que plantea la necesidad de fortalecer el interés por realidades ajenas a uno mismo y por la mejora del entorno próximo.

a) Desarrollo de la iniciativa e impulso de acciones que encierran dificultad y requieren, en consecuencia, la voluntad de tomar responsabilidades y desafios.

El lenguaje coloquial y la fuerza implacable de la economía han arrastrado al concepto emprendedor a tomar una óptica eminentemente económica que ha limitado su significado a este ámbito y ha dejado oculto el significado más extenso del término, que es el que se pretende desarrollar en nuestra asignatura. En este sentido, es de interés conocer el origen del término emprendedor. La lengua expresa el pensamiento y precisar el origen y la evolución del vocablo se plantea como tarea indispensable a la hora de profundizar en el estudio de un concepto e introducirlo en el ámbito educativo. El análisis histórico del término emprender a través de fuentes escritas en español se remonta al 
siglo XI con el sentido de "tomar" (Coromines, 1981). Su incorporación en los diccionarios históricos y etimológicos muestra cómo el sentido del verbo emprender que se ha mantenido a lo largo del tiempo lo identifica con acciones que suponen comenzar o tener iniciativa. Además, tiene una cualidad implícita que lo relaciona con iniciativas que suponen acometer con resolución y superar dificultades (Azqueta, 2019).

La acción de emprender responde a un quehacer propio del ser humano y una tarea específica de su naturaleza. El ser humano no se limita a cubrir necesidades, sino que busca más, anhela más, trabaja para ir más allá, para vencer dificultades y transformar la realidad porque es un ser capaz de metas y fines. El animal no emprende, el hombre sí lo hace, porque tiene capacidad de plantearse metas y elegir con libertad los medios para alcanzar un fin (Segura, 1994). El sentido perfectivo del ser humano lo lleva a emprender, progresar, crear algo diferente, buscar riqueza y bienestar. De esta manera, la educación emprendedora da respuesta a la aspiración natural de la persona al crecimiento y favorece el desarrollo y la mejora de la persona y de la sociedad.

b) La actitud de apertura a lo real que plantea la necesidad de fortalecer el interés por realidades ajenas a uno mismo y por la mejora del entorno próximo.

Se parte de la idea de que el hombre es una existencia abierta hacia dentro y hacia fuera. Es, al mismo tiempo, dependiente e independiente. La sociedad es para el hombre condición de viabilidad porque por sí solo no puede alcanzar la plenitud. Vivir para el ser humano es convivir. La persona es feliz si comparte con los hombres los bienes humanos. Encuentra en la sociedad no solo un medio para cubrir sus necesidades materiales y la búsqueda del bienestar, sino que la sociedad también es ocasión y medio para la perfección de su vida moral. Polo (2003) entiende la persona como coexistencia porque es un ser abierto. En este sentido, el ser humano es "imposible en solitario" (Sellés, 2007, p. 494). El ser humano no solo tiene relaciones, sino que es relación. El tener relaciones supone que estas se pueden tener o no tener, e incluso perderse sin que se produzca una alteración importante; sin embargo, el ser relación es esencial y ontológico a la persona, marca su naturaleza y su identidad personal, 
pertenece a la propia estructura del ser humano (Stein, 1998). El ser humano es un ser abierto al enriquecimiento como persona, puede conocer más y mejor (inteligencia) y puede querer más y mejor (voluntad).

La educación emprendedora posibilita el desarrollo de la apertura personal, abre a la realidad; gracias a ella la persona se comunica y amplía sus relaciones interpersonales. Por medio de la colaboración con los demás en la vida social se realiza la apertura. El aprendizaje y el ejercicio de esa colaboración y solidaridad son manifestaciones de la realización de la apertura, en las que las personas no solo dan, sino que se dan a los demás. La acción educativa manifiesta la esencia de la persona, pero a la vez desvela la coexistencia como distintivo del acto de ser personal. El desarrollo de la apertura constituye una de las principales contribuciones de la educación emprendedora a la sociedad. El crecimiento de la apertura que se funda en la relacionalidad abre a la persona a los demás, fortalece el interés por realidades ajenas a uno mismo a través de la solidaridad y de la búsqueda del bien común. El interés lleva a la acción y a emprender, a acometer acciones por y para los demás, y no solo está motivado por un interés, un bien o un beneficio de orden económico y pecuniario. De esta manera, se fomenta el interés por mejorar el entorno próximo, de la realidad más cercana, y se posibilita el compromiso y la implicación con su realidad social con una actitud proactiva y servicial.

Bajo esta doble perspectiva, a lo largo de la asignatura, se incide en el desarrollo de los indicadores de la competencia emprendedora que hacen referencia a la definición primera del emprendedor y en los que prima la dimensión personal y social frente al carácter económico. Estos indicadores aluden al cultivo de la iniciativa, al desarrollo de la autonomía, al tesón y a la solución de problemas, a la capacidad crítica, al valor de servicio, a la solidaridad, la cooperación social y el pensamiento ético y sostenible. De esta manera se prioriza a la persona y se colabora en el crecimiento de todas las dimensiones del ser personal. Si la educación no tiene en cuenta las necesidades netamente personales, limita el carácter formativo a lo puramente instrumental. Ampliar la significación del emprendimiento se presenta como tarea imprescindible para hacer frente a los desafíos educativos de las próximas décadas y para su inclusión en las aulas. Se requiere una visión holística, integradora, multidimensional y transversal del emprendimiento que evite el reduccionismo economicista (Du Gay, 2004; O’ Rourke, 2014). 


\section{Diseño de la ASIgNATURA Y METOdología de APRENDiZAJE}

El carácter optativo de la asignatura ha permitido el acceso de alumnos procedentes de diferentes facultades, grados e itinerarios formativos. Esta diversidad ha favorecido el perfil interdisciplinar y transversal de la materia, además de enriquecer el trabajo tanto dentro del aula como en las reuniones de seguimiento del proyecto.

De acuerdo con la investigación académica, se considera que las metodologías activas resultan las adecuadas para la enseñanza-aprendizaje del emprendimiento (Paños, 2017; Acosta-García, 2018; Toledo y Sánchez, 2018; Ruiz-Rosa, Gutiérrez-Taño y García-Rodríguez, 2021). En nuestra asignatura se ha empleado el aprendizaje basado en proyectos (ABP), del que se destacan los siguientes aspectos positivos:

1. El proyecto que desarrollan los alumnos no es una actividad complementaria, sino el fundamento de la asignatura (Nieto-Borbor y MartínezSuárez, 2021).

2. Esta metodología permite que los alumnos se organicen por equipos de trabajo. Se requiere que en cada grupo participen alumnos de diferente sexo, procedencias geográficas, grados, facultades e intereses personales. Este modo de agrupamiento facilita la transición de los jóvenes del sistema educativo al ámbito laboral (OCDE, 2015). La disposición para colaborar y las habilidades para trabajar en equipo, para compartir, colaborar y ayudar son algunas de las competencias más demandadas por los empleadores, puesto que resultan imprescindibles para la búsqueda de la mejora continua.

3. El uso del ABP mejora la motivación intrínseca e implicación de los alumnos, pues forman parte activa de su aprendizaje, desarrollan habilidades y actitudes emprendedoras que orientan el aprendizaje hacia la realidad y ponen en práctica lo aprendido. El profesor guía y asesora en el proceso de enseñanza-aprendizaje: conoce el itinerario, el programa de actividades y su interés, se percata de las dificultades del proyecto y aporta, mediante las tutorías de equipo, las orientaciones necesarias para que los alumnos avancen en el desarrollo del proyecto.

4. Aunque existe afinidad entre el aprendizaje basado en proyectos y el aprendizaje por problemas, existen algunas diferencias. El primero 
representa una categoría de aprendizaje más amplia que no se enfoca solo en solucionar un reto, sino que los alumnos toman consciencia de una dificultad, investigan y proponen una solución que no es la única viable, como sucede en el aprendizaje por problemas (Domínguez-Navarro, Carod y Velilla, 2008).

5. Los alumnos tienen que dar respuesta a un desafío o un problema, preferentemente de su entorno próximo, que requiere analizar e investigar el problema, además de planificar y buscar una resolución creativa, factible y realista a esta. La elección del desafío que el profesor presenta a los alumnos es un elemento relevante, pues conviene que despierte la inquietud de los estudiantes hacia problemas reales y actuales.

6. En el aprendizaje resulta de interés no solo el producto final que presentan los alumnos al finalizar el curso a través de su proyecto, sino que también son relevantes en su aprendizaje cada una de las etapas del proyecto, el propio proceso, su desarrollo y la profundidad de cada etapa.

El proyecto debe recoger las principales fases de cualquier proceso emprendedor, como se muestra de manera sintética en la tabla 1 que se presenta a continuación.

TABLA 1

Fases del proyecto emprendedor que desarrollan los alumnos a partir del desafio o problema inicial que presenta el profesor en clase

\begin{tabular}{|l|l|l|l|}
\hline $\begin{array}{c}\text { FASE ANÁLISIS: } \\
\text { Comprensión de la } \\
\text { información }\end{array}$ & $\begin{array}{c}\text { FASE CREATIVA: } \\
\text { Elaboración de nuevas } \\
\text { ideas y soluciones }\end{array}$ & $\begin{array}{c}\text { FASE INNOVADORA: } \\
\text { Llevar las ideas a la } \\
\text { práctica }\end{array}$ & $\begin{array}{c}\text { FASE } \\
\text { EVALUACIÓN: } \\
\text { Valoración de la acción }\end{array}$ \\
\hline $\begin{array}{l}\text { Conocimiento de la } \\
\text { realidad }\end{array}$ & $\begin{array}{l}\text { Planteamiento de } \\
\text { alternativas }\end{array}$ & $\begin{array}{l}\text { Compromiso personal } \\
\text { por la mejora }\end{array}$ & $\begin{array}{l}\text { Resultado de las } \\
\text { acciones realizadas }\end{array}$ \\
\hline $\begin{array}{l}\text { Comprensión y análisis } \\
\text { de la realidad }\end{array}$ & $\begin{array}{l}\text { Evaluación de } \\
\text { alternativas }\end{array}$ & Planificación & $\begin{array}{l}\text { Comparación de } \\
\text { objetivos previstos y } \\
\text { alcanzados }\end{array}$ \\
\hline $\begin{array}{l}\text { Cuestionamiento de la } \\
\text { realidad }\end{array}$ & $\begin{array}{l}\text { Selección de la } \\
\text { alternativa más } \\
\text { adecuada }\end{array}$ & $\begin{array}{l}\text { Actuación y } \\
\text { seguimiento }\end{array}$ & Conclusiones \\
\hline
\end{tabular}

Fuente: elaboración propia 
El profesor acompaña a los equipos de trabajo en cada una de las fases del proyecto y corrige cada entrega. Las clases teóricas se destinan a facilitar a los alumnos los conocimientos y habilidades para desarrollar cada fase del proyecto. De esta manera, los alumnos se familiarizan, entre otras, con las estrategias básicas de generación de ideas y de pensamiento creativo, las metodologías ágiles de innovación y algunas técnicas de comunicación eficaz. A lo largo del cuatrimestre los alumnos realizan entregas parciales y sujetas a los plazos del proyecto. Cada una de estas entregas coincide con las principales fases del proyecto emprendedor, en las que cuentan con la orientación del profesor mediante las tutorías de equipo. El curso finaliza con una feria en la que se exponen y teatralizan los proyectos en formato vídeo (Oparaocha y Daniil, 2020). La evaluación del proyecto dentro de la asignatura tiene una valoración de la mitad de la calificación final de la asignatura.

\section{Evaluación DE LA PROPUESTA}

\subsection{Finalidad de la evaluación, metodología de análisis y procedimiento de trabajo seguido en la evaluación}

El objetivo fundamental de la evaluación ha sido conocer con mayor profundidad cómo han vivido los estudiantes el desarrollo de la asignatura. Se busca mejorar la práctica educativa a través del conocimiento directo de los logros y las debilidades del programa. Esto nos permitirá mejorar la implementación e impacto formativo de la asignatura.

Se ha realizado una evaluación mixta de la asignatura, desarrollada de manera paralela en el tiempo.

En primer lugar, se ha realizado una evaluación cuantitativa a través de una encuesta que responde a seis temas de análisis que se consideran de interés para la asignatura. La selección de estas categorías surgió, en primer lugar, fruto de la voluntad de la docente que tenía interés por recoger el feedback de los alumnos y, en segundo lugar, de manera inductiva, a partir de las primeras conversaciones con los estudiantes al inicio del cuatrimestre. Las seis categorías analizadas han sido las siguientes: 1) grado de satisfacción general con 
la asignatura; 2) metodología didáctica; 3) correspondencia entre las horas de trabajo de dedicación y los créditos ECTS que tiene asignada la asignatura; 4) la coordinación entre la parte teórica de la asignatura y el proyecto; 5) la forma de evaluación de la asignatura, y 6) el interés para la adquisición de competencias para la formación del alumno. Los encuestados responden de manera anómina, mediante un formulario en línea. Para la valoración de cada uno de los items, se utiliza la escala de Likert, del 1 al 5, siendo el 1 la expresión de la minima satisfacción y el 5 la máxima.

En segundo lugar, se aplica la metodología cualitativa (Flick, 2015) a través de entrevistas semiestructuradas. Las conversaciones las mantuvo la propia docente. La información resultante de las conversaciones personales con los alumnos se ha organizado, de manera inductiva y manual, en las mismas 6 categorías de análisis que se han empleado en la categorización de las respuestas a la encuesta y que se indican en el párrafo anterior. El resto de información se ha desechado.

Se considera apropiado combinar esta doble metodología porque reporta equilibrio a la investigación. El análisis cuantitativo aporta objetividad y este se enriquece con el análisis cualitativo a través de las entrevistas semiestructuradas que proporcionan profundidad (Kvale, 2011) y facilita llegar a conclusiones concretas.

\subsection{Selección de la muestra}

La muestra ha quedado conformada de la siguiente manera:

- En la encuesta han participado veintiocho estudiantes que han cursado la asignatura y que, voluntariamente, antes del examen teórico de la asignatura accedieron a participar en la evaluación y respondieron a la encuesta. Los alumnos cursaban: $4 .^{\circ}$ curso del grado de Psicología, especialidad Empresa, que pertenecen a la Facultad de Educación y Psicología; 3. ${ }^{\text {er }}$ y $4{ }^{\circ}$ curso del grado de Administración y Dirección de Empresa de la Facultad de Ciencias Económicas y Empresariales; $4^{\circ}$ curso del grado de Management Assistant (actual grado en Applied Management) de la 
Facultad ISSA School of Applied Management, y alumnos internacionales de $1 .^{\text {er }}$ año que cursan el International Foundation Program.

- Las entrevistas semiestructuradas fueron individuales y se realizaron a 5 alumnos: un alumno por cada grado o curso (1 alumna de Psicología Empresa; 2 alumnos de ADE; 1 de $3 .^{\circ}$ y otro de $4 .^{\circ}$ curso; 1 alumna de 4. ${ }^{\circ}$ de Management Assistant, y 1 alumna de $1 .^{\text {er }}$ curso del Programa Internacional).

\subsection{Resultados de la evaluación de la asignatura}

a) Análisis cuantitativo

A continuación, se muestran, en el siguiente gráfico de red (figura 1), los resultados de la encuesta en la que se abordan 6 temas relevantes para el desarrollo de la asignatura. Los resultados que recoge la figura 1 muestran la media de la puntuación de cada uno de los ítems del cuestionario.

\begin{tabular}{|l|c|c|c|c|c|c|c|}
\hline & P1 & P2 & P3 & P4 & P5 & P6 & Media \\
\hline $\begin{array}{l}\text { Formación para emprendimiento } \\
\text { e innovación }\end{array}$ & 3,9 & 4,3 & 3,8 & 4,3 & 4,3 & 3,7 & 4,1 \\
\hline
\end{tabular}

El análisis concede los valores más altos a la metodología, la coordinación de la teoría que se explica en clase y el proyecto que presentan los alumnos y el sistema de evaluación de la asignatura. Sin embargo, los alumnos señalan la falta de correspondencia entre las horas de trabajo que requiere la asignatura y los créditos ECTS que tiene asignada y la valoración que se otorga a las competencias que reporta esta materia para la propia formación. 
FIGURA 1

Resultados de evaluación según las seis categorías de análisis

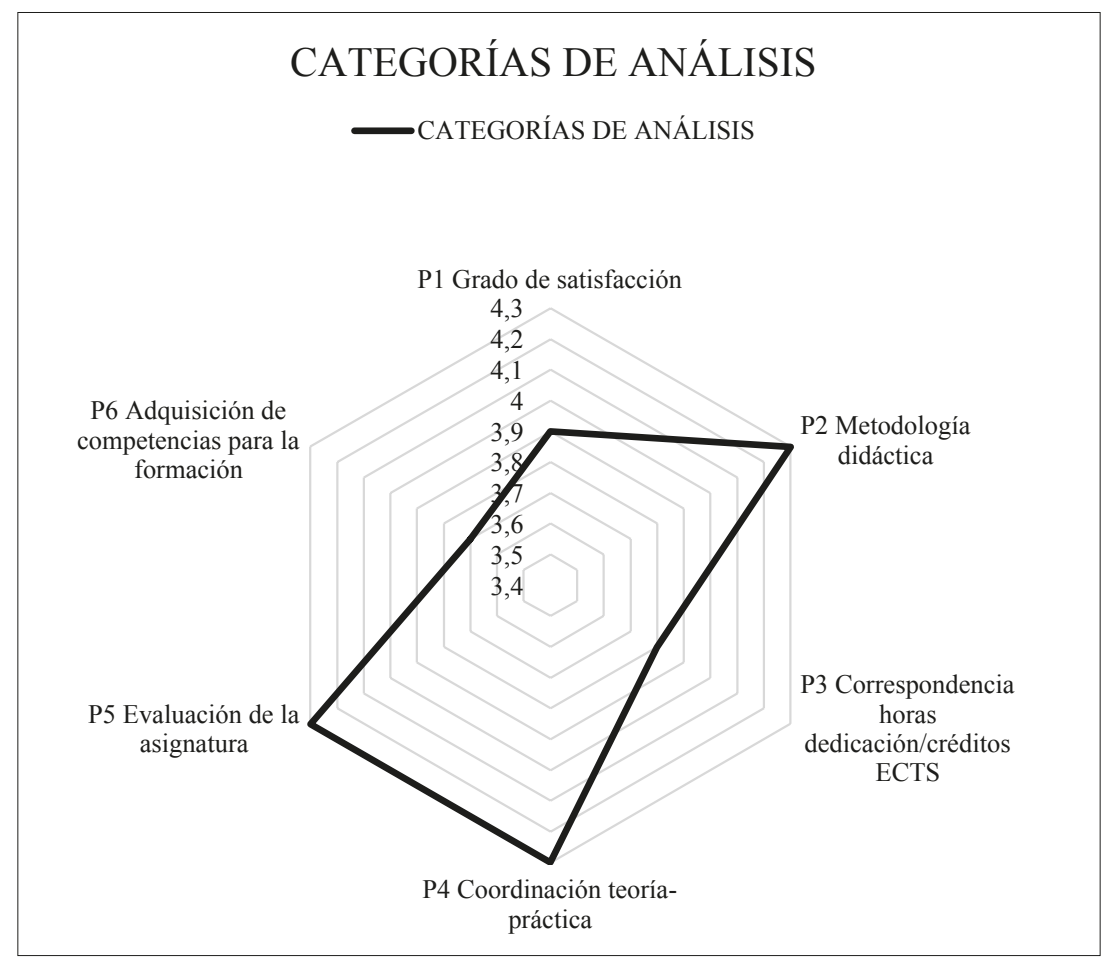

Fuente: elaboración propia

b) Análisis cualitativo

El análisis cualitativo del contenido de las entrevistas clarifica los datos numéricos que arroja el análisis cuantitativo. Los datos que los participantes han ofrecido mediante las entrevistas se han distribuido, de manera manual, en las 6 categorías de análisis. Cada una de esas categorías ha generado una serie de dilemas a partir de los cuales se podrán deducir los aspectos fuertes y débiles de la asignatura. En la tabla 2, se presenta un resumen que recoge los principales dilemas de cada temática. 
TABLA 2

Principales dilemas que han surgido en torno a las 6 categorías de análisis

\begin{tabular}{|l|l|}
\hline Grado de satisfacción general & $\begin{array}{l}\text { - Desigual interés por parte de los alumnos. } \\
\text { - Se valora su carácter práctico. }\end{array}$ \\
\hline Metodología didáctica & $\begin{array}{l}\text { - Recogida de los puntos de vista de los alumnos. } \\
\text { - El proyecto aborda los temas esenciales de la asignatura. } \\
\text { - Dificultades para el trabajo en grupo tanto dentro como fuera } \\
\text { del aula. }\end{array}$ \\
\hline $\begin{array}{l}\text { Correspondencia horas dedicación/ } / \text { Diferentes niveles de implicación por parte de los alumnos } \\
\text { en el proyecto. }\end{array}$ \\
\hline créditos ECTS & $\begin{array}{l}\text { - Las horas de dedicación al proyecto son superiores a los } 3 \\
\text { créditos asignados. }\end{array}$ \\
\hline Coordinación contenidos & $\begin{array}{l}\text { - Aplicabilidad de la teoría en la práctica del proyecto. } \\
\text { - Se requiere coordinación temporal entre la explicación teórica } \\
\text { en el aula con las fases del proyecto. }\end{array}$ \\
\hline Evaluación de la asignatura & $\begin{array}{l}\text { - Valoración de la necesidad del examen teórico. } \\
\text { - Dificultad para calificar individualmente a cada alumno en } \\
\text { un proyecto que supone trabajar en equipo. }\end{array}$ \\
\hline $\begin{array}{l}\text { Adquisición de competencias para la } \\
\text { formación }\end{array}$ & - Impacto en la formación en función de la Facultad de origen. \\
\hline
\end{tabular}

Fuente: elaboración propia

El análisis cualitativo reporta un mayor número de ideas y sugerencias relativos a los aspectos metodológicos y de evaluación de la asignatura que sin embargo tienen una calificación más alta en los resultados de la encuesta.

En cuanto a las limitaciones del estudio, hay que indicar que se trata de una evaluación interna en un único curso académico y en la que solo han participado una parte de los alumnos matriculados en la asignatura. Se requieren evaluaciones en futuras convocatorias que permitan generalizar algunos resultados e impulsar mejoras de mayor calado en la asignatura.

\subsection{Discusión de resultados de la evaluación de la asignatura}

El análisis de los tópicos ha permitido deducir algunas cuestiones que se exponen a continuación: 
1. Se valora la asignatura fundamentalmente por su carácter práctico y esfuerzo moderado. No es una asignatura difícil de comprender ni requiere memorizar.

2. Respecto a la metodología del trabajo por proyectos, se valora positivamente porque plantea un escenario educativo diferente al que los alumnos están habituados: se aprende la teoría a través de la práctica, permite desarrollar la iniciativa y dar cauce a las ideas personales. Sin embargo, el mayor reto para los alumnos es hacer frente a las dificultades que supone el trabajo en equipo. En esta línea, los alumnos mencionan expresamente como dificultades para el trabajo en equipo los siguientes aspectos: la coordinación de alumnos de diferentes grados, la asistencia a clase intermitente o los diferentes grados de motivación personal que afectan a la implicación, el trabajo y los posibles resultados del proyecto. Los alumnos perciben el trabajo en equipo como una dificultad o un requisito estresante más que como un recurso de aprendizaje.

3. Los resultados señalan un aumento importante en la percepción de la carga de trabajo que supone para los alumnos el trabajo por proyectos frente al sistema tradicional de clases. Esta situación plantea dos cuestiones. En primer lugar, la necesidad de revisar las horas de dedicación del proyecto dentro de la asignatura y los créditos que tiene asignados (en la actualidad 3 créditos ECTS). En segundo lugar, se requiere mejorar y familiarizar a los alumnos en las competencias propias del trabajo en equipo, la coordinación y comunicación en el equipo además de la planificación, gestión y distribución del tiempo a lo largo del cuatrimestre.

4. La visión de la asignatura por parte de los alumnos es positiva en lo relativo a la coordinación entre la parte teórica y práctica de la asignatura y la aplicabilidad de lo explicado en clase. Consideran que esta no se limita a acumular conocimiento. Se trata de una materia que genera aprendizajes con bastante nivel de autonomía por parte de los alumnos, puesto que permite experimentar y practicar, además de promover la toma de decisiones a lo largo del proyecto. Sin embargo, se requiere sincronizar y planificar mejor la explicación de la teoría con la parte práctica.

5. Aunque, en la encuesta, los alumnos valoran positivamente el sistema de evaluación de la asignatura, sin embargo, las entrevistas personales muestras ciertas discrepancias con relación al sistema de evaluación 
de la asignatura. Un primer aspecto hace referencia a la posibilidad de prescindir de la evaluación teórica de la asignatura, dando absoluto protagonismo al proyecto dentro de la materia. Un segundo elemento plantea el reto de la evaluación no solo de la calidad del trabajo que los alumnos entregan al finalizar la asignatura, sino también la necesidad de valorar el trabajo individual de cada alumno dentro del proyecto de equipo. Ambos dilemas implican decisiones y cambios relevantes.

6. Respecto a la adquisición de competencias para la formación del alumno se estima diferente valoración del impacto en la propia formación en función de la facultad de origen. Los alumnos vinculados al ámbito empresarial, como son los alumnos de la Facultad de Empresa y los de Psicología especialidad Empresa, valoran especialmente esta asignatura. Es menor el interés de los alumnos procedentes de otros grados.

A partir de estas cuestiones se podrán desarrollar, a modo de conclusión, algunas propuestas de mejoras en la asignatura, tanto a corto como a medio plazo.

\section{Conclusiones}

El análisis ha permitido conocer los aspectos fuertes y débiles de la asignatura. Entre los primeros se valora su marcado carácter práctico y el empleo de la metodología por proyectos. Respecto a las debilidades se considera que se pueden introducir algunas mejoras con dos niveles de concreción, corto y medio plazo. Las primeras se podrían implementar el próximo curso académico. Las segundas requieren próximas evaluaciones que ratifiquen las valoraciones porque comprometen la memoria del título y son, por tanto, mejoras que se podrían implementar a medio plazo.

En líneas generales, se plantean las siguientes mejoras:

- Fortalecer la adquisición de las competencias que favorezcan el desempeño de los estudiantes para el trabajo en equipo. En concreto, la mejora en las habilidades para la planificación del tiempo, la adquisición de responsabilidades compartidas, la cohesión del grupo y la comunicación ágil y eficaz. 
- Convendría revisar el modo de evaluación del trabajo por proyectos y que se valore el trabajo individual de cada alumno dentro del proyecto de equipo. En este sentido, el enfoque conocido como "caja blanca", a semejanza de la empleada (Fidalgo-Blanco, Lerís, Sein-Echaluce y García-Peñalvo, 2013), puede ser una opción viable. El profesor valora, entre otros aspectos, la planificación, el reparto de tareas, la coordinación, las responsabilidades asumidas por cada miembro del equipo, etc. A medio plazo, se podría valorar la posibilidad de prescindir de la evaluación teórica de la asignatura dando absoluto protagonismo al proyecto dentro de la asignatura puesto que el carácter práctico de la materia posibilita poner en práctica lo aprendido.

- Se ve necesario revisar las horas de dedicación del proyecto dentro de la asignatura y los créditos que tiene asignados (en la actualidad 3 créditos ECTS), y valorar si, dado el carácter aplicado de la asignatura, el valor del proyecto frente a la teoría tiene que ser mayor.

- Es precisa una planificación exquisita para que la parte teórica y práctica se complementen y se dote a los estudiantes de los conocimientos y habilidades que requieren para desarrollar con éxito cada una de las fases del proyecto.

Finalmente, la experiencia de evaluación de esta materia ha resultado positiva: llevar a cabo evaluaciones internas de la asignatura complementa las estimaciones que, de manera habitual, realizan los órganos de evaluación de la calidad de la docencia de la propia universidad. La evaluación interna es menos generalista y más profunda; ayuda al docente a repensar algunos aspectos de la asignatura y permite impulsar mejoras con mayor agilidad.

\section{Agradecimientos}

Esta publicación está enmarcada dentro del Proyecto "Formación del Potencial Emprendedor. Generación de un Modelo Educativo de Identidad Emprendedora", con Referencia PID2019-104408GB-I00, dentro del Plan Estatal 2017-2020 de Proyectos I+D+i (Generación de Conocimiento), financiado 
por el Ministerio de Ciencia e Innovación - Agencia Estatal de Investigación / PID2019-104408GB-I00/AEI/10.13039/501100011033

\section{BiBLIOGRAFÍA}

Acosta-García, A. (2018). Cómo se forma un emprendedor: El aprendizaje basado en la gestión de proyectos. Debates IESA, volumen XXIII, número 3 (julio-septiembre). Recuperado de: https://bit.ly/3x57pSI

Amilburu, M., Ruiz-Corbella, M. y García-Gutiérrez, J. (2017). La formación en capacidades en la educación superior: la responsabilidad social en la universidad, en J.A. Ibañez y J.L. Fuentes (eds.). Educación y capacidades, hacia un nuevo enfoque del desarrollo humano (pp. 175-186). Dykinson

Audretsch, D.B. (2014). From the entrepreneurial univer-sity to the university for the entrepreneurial society. Journal of Technology Transfer 39, 313-321. AzQueTA, A. (2019). Análisis del concepto “emprendedor" y su incorporación al ámbito educativo. Teoría de la Educación. Revista Interuniversitaria 31(1), 57-80. DOI: https://doi.org/10.14201/teri.19756.

AzQueta, A. y NAval, C. (2019). Educación para el emprendimiento. Revista española de pedagogía 77(274), 517-534. DOI: https://doi.org/10.22550/ REP77-3-2019-03.

Bernal Guerrero, A. y CÁrdenas, A. (2017). Evaluación del potencial emprendedor en escolares. Una investigación longitudinal. Educación XX1 20(2), 73-94. DOI: https://doi.org/10.5944/educxx1.19032.

Castro, J., Barrenechea, J. e Ibarra, A. (2011). Cultura emprendedora, innovación y competencias en la Educación Superior. El caso del Programa GAZE. Arbor 187 (extra 3), 207-212.

Colther, C. M., Fecci, E., Cayun, G. y Rojas, J. (2020). Enseñanza de la cultura emprendedora en la Universidad. El caso de la Universidad Austral de Chile. Formación Universitaria 13(4), 129-138. DOI: https://doi.org/10.4067/ S0718-50062020000400129.

Comisión Europea (2011). Comunicación de la Comisión al Parlamento Europeo, al Consejo, al Comité Económico y Social Europeo y al Comité de las Regiones. Apoyar el crecimiento y el empleo - una agenda para la 
modernización de los sistemas de educación superior en Europa. COM (2011) 567 final, del 20 de septiembre de 2011.

Conferencia de Rectores de las Universidades Españolas-CRUE- (2018). Declaración de Salamanca. IV Encuentro Internacional Universia de Rectores, Salamanca, 21 y 22 de mayo de 2018. Recuperado: de: https://cutt. ly/7xB7JIq

Consejo Europeo (2000). Conclusiones de la Presidencia. Consejo Europeo de Lisboa 23 y 24 de marzo 2000.

Coromines, J. (1981). Breve diccionario etimológico de la lengua castellana. Gredos.

Domínguez-Navarro, J., Carod, E. y Velilla, M. ${ }^{\text {a }-J . ~(2008) . ~ C o m p a r a t i v a ~ e n t r e ~}$ el aprendizaje basado en proyectos y el aprendizaje basado en problemas. II Jornadas de Innovación Docente, Tecnologías de la Información y de la Comunicación e Investigación Educativa. Universidad de Zaragoza.

Du GAY, P. (2004). Against "Enterprise" (but not against "enterprise", for that would make no sense). Organization 11(1), 37-57.

Espejo, B. (2001). Políticas educativas para el nuevo siglo. Hespérides.

EUROPEAN COMISSION (2008). Entrepreneurship in higher education, especially in non-business studies. European Comission.

EURYDICE (2016). La educación para el emprendimiento en los centros educativos en Europa. Oficina publicaciones Unión Europea.

Flick, U. (2015). El diseño de la investigación cualitativa. Editorial Morata.

Fidalgo-Blanco, Á., Lerís, D., Sein-Echaluce, M. L. y García-Peñalvo, F. J. (2013). Indicadores para el seguimiento y evaluación de la competencia de trabajo en equipo a través del método CTMTC. II Congreso Internacional sobre Aprendizaje, Innovación y Competitividad (CINAIC 2013). Madrid, 6-8 de noviembre de 2013.

García del Dujo, A. (2011). Los límites de la Educación. Revista portuguesa de pedagogía (extra-serie), 181-194.

GILl, R. (2014). If you're struggling to survive day-to-day': class optimism and contradiction in entrepreneurial discourse. Organization 21(1), 50-67. https://doi.org/10.1177/1350508412464895

GonzÁlez-Tejerina, S. y Vieira, M.-J. (2020). La formación en emprendimiento en Educación Primaria y Secundaria: una revision sistemática. Revis- 
ta Complutense de Educación 32(1), 99-111. DOI: https://doi.org/10.5209/ rced.68073.

Hervás-Oliver, J. L., Boronat, C. y Messana, I. (2017). La universidad española como plataforma de emprendimiento: hacia la universidad emprendedora del futuro. Economía industrial 404, 11-19.

HitTy, U. y O'Gorman, C. (2004). What is ‘enterprise education'? An analysis of the objectives and methods of enterprise education programmes in four European countries, Education + Training 46(1), 11-23. DOI: https://doi. org/10.1108/00400910410518188.

Komulainen, K., Naskali, P., Korhonen, M. y Keskitalo-Foley, S. (2011). Internal entrepreneurship-a Trojan horse of the neoliberal governance of education? Finnish pre-and in-service teachers' implementation of and resistance towards entrepreneurship education. Journal for Critical Education Policy Studies 9(1), 341-374.

Kvale, S. (2011). Las entrevistas en investigación cualitativa. Editorial Morata.

Martínez-Clares, P.; González-Lorente, C. y Rebollo, N. (2019). Competencias para la empleabilidad: un modelo de ecuaciones estructurales en la Facultad de Educación. Revista de Investigación Educativa 37(1), 57-73. Recuperado de: http://dx.doi.org/10.6018/rie.37.1.343891

Nieto-Borbor, C. L. y Martínez-Suárez, P. C. (2021). Caracterización del aprendizaje basado en proyectos para el fortalecimiento de competencias emprendedoras. Polo del Conocimiento 6(3), 2482-2499.

Nussbaum, M. (2012). Crear capacidades. Propuesta para el desarrollo humano. Paidós.

OCDE (2015). Skills Strategy Informe de diagnóstico de la estrategia de competencias de la OCDE. Resumen. Directorate for Education and Skills OECD.

Oparaocha, G. O. y Danill, P. (2020). Theatricalization of enterprise education: A call or "action". Arts and Humanities in Higher Education 19(1), 20-35. Recuperado de: https://doi.org/10.1177/1474022218793268

O'Rourke, B. K. (2014). Learning from interacting: language, economics and the entrepreneur. On the Horizon 22(4), 245-255.

PAÑos, J. (2017). Educación emprendedora y metodologías activas para su fomento. Revista Electrónica Interuniversitaria de Formación del Profesorado 20(3), 33-48. DOI: https://doi.org/10.6018/reifop.20.3.272221. 
Polo, L. (2003). Antropología transcendental. Editorial Universidad de Navarra.

PRING, R. (2016). Una filosofía de la educación políticamente incómoda. Narcea.

Rome Ministerial (2020). Communiqué. European Higher Education Area. Recuperado de: https://cutt.ly/ZnrpXx5 (consulta: 19/11/2020).

Rosendahl, L., Sloof, R. y Van PraAg, M. (2014). The Effect of Early Entrepreneurship Education: Evidence from a Field Experiment. European Economic Review 72, 76-97. DOI: https://doi.org/10.1016/j.euroecorev.2014.09.002.

Ruiz-Herrero, J. A. (2013). Nuevos espacios productivos en el capitalismo cognitivo: el ejemplo del emprendimiento. XI Congreso Español de Sociología, Federación Española de Sociología. Madrid, 10-12 de julio de 2013.

Ruiz-Rosa, I., Gutiérrez-TAÑo, D. y García-Rodríguez, F. J. (2021). El Aprendizaje Basado en Proyectos como herramienta para potenciar la competencia emprendedora). Cultura y Educación 33(2), 316-344. DOI: https://doi.org/ 10.1080/11356405.2021.1904657.

Schultz, T. (1961). Investment in Human Capital. The American Economic Review 51(1), 1-17.

Segura, A. (1994). La inserción de la Persona en la Empresa. Cuadernos Empresa Humanismo 41, 3-31.

SElLÉs, J. (2007). Antropología para inconformes. Una antropología abierta al futuro. Universidad de Navarra.

SEN, A. (1985). Well-Being, Agency, and Freedom: The Dewey Lectures 1984. Journal of Philosophy 82, 169-221.

Simon M. y Masschelein, J. (2008). The gobernmentalization of learning and the assemblage of a learning apparatus. Educational Theory 58(4), 391-415.

SteIn, E. (1998). La estructura de la persona humana. Biblioteca de Autores Cristianos.

Toledo, P. y Sánchez, J. M. (2018). Aprendizaje basado en proyectos: una experiencia universitaria. Profesorado. Revista de Currículum y Formación de Profesorado 22(2), 471-491. DOI: https://orcid.org/0000-0002-2160-0000. 